\title{
First Measurement of Timelike Compton Scattering
}

P. Chatagnon $\odot,{ }^{20, *}$ S. Niccolai, ${ }^{20}$ S. Stepanyan, ${ }^{36}$ M. J. Amaryan, ${ }^{29}$ G. Angelini, ${ }^{12}$ W. R. Armstrong, ${ }^{1}$ H. Atac, ${ }^{35}$ C. Ayerbe Gayoso, ${ }^{44, \dagger}$ N. A. Baltzell, ${ }^{36}$ L. Barion, ${ }^{13}$ M. Bashkanov, ${ }^{42}$ M. Battaglieri, ${ }^{36,15}$ I. Bedlinskiy, ${ }^{25}$ F. Benmokhtar, ${ }^{7}$ A. Bianconi, ${ }^{39,19}$ L. Biondo, ${ }^{15,18,40}$ A. S. Biselli, ${ }^{8}$ M. Bondi,${ }^{15}$ F. Bossù, ${ }^{3}$ S. Boiarinov, ${ }^{36}$ W. J. Briscoe, ${ }^{12}$ W. K. Brooks,${ }^{37,36}$ D. Bulumulla, ${ }^{29}$ V. D. Burkert, ${ }^{36}$ D. S. Carman, ${ }^{36}$ J. C. Carvajal, ${ }^{10}$ M. Caudron,${ }^{20}$ A. Celentano, ${ }^{15}$ T. Chetry, ${ }^{24,28}$ G. Ciullo, ${ }^{13,9}$ L. Clark, ${ }^{41}$ P. L. Cole,${ }^{22}$ M. Contalbrigo, ${ }^{13}$ G. Costantini, ${ }^{39,19}$ V. Crede, ${ }^{11}$ A. D'Angelo, ${ }^{16,32}$ N. Dashyan, ${ }^{45}$ M. Defurne,${ }^{3}$ R. De Vita, ${ }^{15}$ A. Deur, ${ }^{36}$ S. Diehl,${ }^{30,5}$ C. Djalali, ${ }^{28}$ R. Dupré,${ }^{20}$ H. Egiyan, ${ }^{36}$ M. Ehrhart, ${ }^{20,}$ A. El Alaoui, ${ }^{37}$ L. El Fassi, ${ }^{24}$ L. Elouadrhiri, ${ }^{36}$ S. Fegan, ${ }^{42}$ R. Fersch, ${ }^{4}$ A. Filippi,${ }^{17}$ G. Gavalian, ${ }^{36}$ Y. Ghandilyan, ${ }^{45}$ G. P. Gilfoyle, ${ }^{31}$ F. X. Girod, ${ }^{36}$ D. I. Glazier, ${ }^{41}$ A. A. Golubenko, ${ }^{33}$ R. W. Gothe, ${ }^{34}$ Y. Gotra,${ }^{36}$ K. A. Griffioen,${ }^{44}$ M. Guidal,${ }^{20}$ L. Guo, ${ }^{10}$ H. Hakobyan, ${ }^{37,45}$ M. Hattawy, ${ }^{29}$ T. B. Hayward, ${ }^{5,44}$ D. Heddle, ${ }^{4,36}$ A. Hobart, ${ }^{20}$ M. Holtrop, ${ }^{26}$ C. E. Hyde, ${ }^{29}$ Y. Ilieva, ${ }^{34}$ D. G. Ireland, ${ }^{41}$ E. L. Isupov, ${ }^{33}$ H. S. Jo, ${ }^{21}$ K. Joo, ${ }^{5}$ M. L. Kabir, ${ }^{24}$ D. Keller, ${ }^{43}$ G. Khachatryan, ${ }^{45}$ A. Khanal, ${ }^{10}$ A. Kim, ${ }^{5}$ W. Kim, ${ }^{21}$ A. Kripko, ${ }^{30}$ V. Kubarovsky ${ }^{36}$ S. E. Kuhn, ${ }^{29}$ L. Lanza, ${ }^{16}$ M. Leali, ${ }^{39,19}$ S. Lee, ${ }^{23}$ P. Lenisa, ${ }^{13,9}$ K. Livingston, ${ }^{41}$ I. J. D. MacGregor, ${ }^{41}$ D. Marchand,${ }^{20}$ L. Marsicano, ${ }^{15}$ V. Mascagna,,${ }^{38,19,8}$ B. McKinnon, ${ }^{41}$ C. McLauchlin, ${ }^{34}$ S. Migliorati, ${ }^{39,19}$ M. Mirazita, ${ }^{14}$ V. Mokeev,${ }^{36}$ R. A. Montgomery,${ }^{41}$ C. Munoz Camacho,${ }^{20}$ P. Nadel-Turonski, ${ }^{36}$ P. Naidoo, ${ }^{41}$ K. Neupane, ${ }^{34}$ T. R. O’Connell, ${ }^{5}$ M. Osipenko, ${ }^{15}$ M. Ouillon, ${ }^{20}$ P. Pandey, ${ }^{29}$ M. Paolone,${ }^{27,35}$

L. L. Pappalardo, ${ }^{13,9}$ R. Paremuzyan,${ }^{36,26}$ E. Pasyuk, ${ }^{36}$ W. Phelps,${ }^{4,12}$ O. Pogorelko, ${ }^{25}$ J. Poudel, ${ }^{29}$ J. W. Price, ${ }^{2}$ Y. Prok,${ }^{29}$ B. A. Raue, ${ }^{10}$ T. Reed, ${ }^{10}$ M. Ripani, ${ }^{15}$ A. Rizzo, ${ }^{16,32}$ P. Rossi, ${ }^{36}$ J. Rowley, ${ }^{28}$ F. Sabatié, ${ }^{3}$ A. Schmidt, ${ }^{12}$ E. P. Segarra ${ }^{23}$ Y. G. Sharabian, ${ }^{36}$ E. V. Shirokov, ${ }^{33}$ U. Shrestha, ${ }^{5,28}$ D. Sokhan, ${ }^{3,41}$ O. Soto, ${ }^{14,37}$ N. Sparveris, ${ }^{35}$ I. I. Strakovsky, ${ }^{12}$ S. Strauch, ${ }^{34}$ N. Tyler, ${ }^{34}$ R. Tyson, ${ }^{41}$ M. Ungaro, ${ }^{36}$ S. Vallarino, ${ }^{13}$ L. Venturelli, ${ }^{39,19}$ H. Voskanyan, ${ }^{45}$ A. Vossen, ${ }^{6,36}$ E. Voutier, ${ }^{20}$ D. P. Watts, ${ }^{42}$ K. Wei, ${ }^{5}$ X. Wei,${ }^{36}$ R. Wishart, ${ }^{41}$ B. Yale, ${ }^{44}$ N. Zachariou, ${ }^{42}$ J. Zhang, ${ }^{43}$ and Z. W. Zhao ${ }^{6}$

\section{(CLAS Collaboration)}

\author{
${ }^{1}$ Argonne National Laboratory, Argonne, Illinois 60439, USA \\ ${ }^{2}$ California State University, Dominguez, Hills, Carson, California 90747, USA \\ ${ }^{3}$ IRFU, CEA, Université Paris-Saclay, F-91191 Gif-sur-Yvette, France \\ ${ }^{4}$ Christopher Newport University, Newport News, Virginia 23606, USA \\ ${ }^{5}$ University of Connecticut, Storrs, Connecticut 06269, USA \\ ${ }^{6}$ Duke University, Durham, North Carolina 27708-0305, USA \\ ${ }^{7}$ Duquesne University, 600 Forbes Avenue, Pittsburgh, Pennsylvania 15282, USA \\ ${ }^{8}$ Fairfield University, Fairfield, Connecticut 06824, USA \\ ${ }^{9}$ Universita' di Ferrara, 44121 Ferrara, Italy \\ ${ }^{10}$ Florida International University, Miami, Florida 33199, USA \\ ${ }^{11}$ Florida State University, Tallahassee, Florida 32306, USA \\ ${ }^{12}$ The George Washington University, Washington, D.C. 20052, USA \\ ${ }^{13}$ INFN, Sezione di Ferrara, 44100 Ferrara, Italy \\ ${ }^{14}$ INFN, Laboratori Nazionali di Frascati, 00044 Frascati, Italy \\ ${ }^{15}$ INFN, Sezione di Genova, 16146 Genova, Italy \\ ${ }^{16}$ INFN, Sezione di Roma Tor Vergata, 00133 Rome, Italy \\ ${ }^{17}$ INFN, Sezione di Torino, 10125 Torino, Italy \\ ${ }^{18}$ INFN, Sezione di Catania, 95123 Catania, Italy \\ ${ }^{19}$ INFN, Sezione di Pavia, 27100 Pavia, Italy \\ ${ }^{20}$ Université Paris-Saclay, CNRS/IN2P3, IJCLab, 91405 Orsay, France \\ ${ }^{21}$ Kyungpook National University, Daegu 41566, Republic of Korea \\ ${ }^{22}$ Lamar University, 4400 M. L. King, Jr. Boulevard, P.O. Box 10046, Beaumont, Texas 77710, USA \\ ${ }^{23}$ Massachusetts Institute of Technology, Cambridge, Massachusetts 02139-4307, USA \\ ${ }^{24}$ Mississippi State University, Mississippi State, Mississippi 39762-5167, USA \\ ${ }^{25}$ National Research Centre Kurchatov Institute_ITEP, Moscow, 117259, Russia \\ ${ }^{26}$ University of New Hampshire, Durham, New Hampshire 03824-3568, USA \\ ${ }^{27}$ New Mexico State University, P.O. Box 30001, Las Cruces, New Mexico 88003, USA \\ ${ }^{28}$ Ohio University, Athens, Ohio 45701, USA \\ ${ }^{29}$ Old Dominion University, Norfolk, Virginia 23529, USA \\ ${ }^{30}$ II Physikalisches Institut der Universitaet Giessen, 35392 Giessen, Germany
}


${ }^{31}$ University of Richmond, Richmond, Virginia 23173, USA

${ }^{32}$ Universita' di Roma Tor Vergata, 00133 Rome, Italy

${ }^{33}$ Skobeltsyn Institute of Nuclear Physics, Lomonosov Moscow State University, 119234 Moscow, Russia

${ }^{34}$ University of South Carolina, Columbia, South Carolina 29208, USA

${ }^{35}$ Temple University, Philadelphia, Pennsylvania 19122, USA

${ }^{36}$ Thomas Jefferson National Accelerator Facility, Newport News, Virginia 23606, USA

${ }^{37}$ Universidad Técnica Federico Santa María, Casilla 110-V Valparaíso, Chile

${ }^{38}$ Università degli Studi dell'Insubria, 22100 Como, Italy

${ }^{39}$ Università degli Studi di Brescia, 25123 Brescia, Italy

${ }^{40}$ Universit'a degli Studi di Messina, 98166 Messina, Italy

${ }^{41}$ University of Glasgow, Glasgow G12 8QQ, United Kingdom

${ }^{42}$ University of York, York YO10 5DD, United Kingdom

${ }^{43}$ University of Virginia, Charlottesville, Virginia 22901, USA

${ }^{44}$ College of William and Mary, Williamsburg, Virginia 23187-8795, USA

${ }^{45}$ Yerevan Physics Institute, 375036 Yerevan, Armenia

(Received 31 August 2021; revised 21 October 2021; accepted 11 November 2021; published 22 December 2021)

We present the first measurement of the timelike Compton scattering process, $\gamma p \rightarrow p^{\prime} \gamma^{*}\left(\gamma^{*} \rightarrow e^{+} e^{-}\right)$, obtained with the CLAS12 detector at Jefferson Lab. The photon beam polarization and the decay lepton angular asymmetries are reported in the range of timelike photon virtualities $2.25<Q^{\prime 2}<9 \mathrm{GeV}^{2}$, squared momentum transferred $0.1<-t<0.8 \mathrm{GeV}^{2}$, and average total center-of-mass energy squared $s=14.5 \mathrm{GeV}^{2}$. The photon beam polarization asymmetry, similar to the beam-spin asymmetry in deep virtual Compton scattering, is sensitive to the imaginary part of the Compton form factors and provides a way to test the universality of the generalized parton distributions. The angular asymmetry of the decay leptons accesses the real part of the Compton form factors and thus the $D$-term in the parametrization of the generalized parton distributions.

DOI: 10.1103/PhysRevLett.127.262501

Most of the mass of the observable universe comes from protons and neutrons. The nucleon mass comes mainly from the interactions between their fundamental constituents, the quarks and the gluons (or "partons"), which are described by the quantum chromodynamics (QCD) Lagrangian [1]. However, QCD-based calculations cannot yet be performed to fully explain the properties of nucleons in terms of their constituents. Phenomenological functions are used to connect experimental observables with the QCD matrix elements describing the dynamics of partons in nucleons. Typical examples of such functions are datadriven parametrizations for the form factors (FFs) and parton distribution functions (PDFs). Generalized parton distributions (GPDs) combine and extend the information contained in FFs and PDFs [2]. They describe the correlations between the longitudinal momentum and transverse spatial position of the partons inside the nucleon, giving access to the contribution of the orbital momentum of the quarks to the nucleon, and they are sensitive to the correlated $q-\bar{q}$ components [3-8].

Published by the American Physical Society under the terms of the Creative Commons Attribution 4.0 International license. Further distribution of this work must maintain attribution to the author(s) and the published article's title, journal citation, and DOI. Funded by SCOAP.
Compton scattering has long been identified as a golden process among deep exclusive reactions to study GPDs experimentally. Deep virtual Compton scattering (DVCS), the exclusive electroproduction of a real photon $\left(e p \rightarrow e^{\prime} p^{\prime} \gamma\right)$, proposed in Refs. [4-6], has been the preferred tool for accessing GPDs until now [9-14]. Meanwhile, timelike Compton scattering (TCS) has been widely discussed theoretically [15-18], but never measured experimentally. This Letter reports on the first measurement of TCS on the proton, $\gamma p \rightarrow p^{\prime} \gamma^{*}\left(\gamma^{*} \rightarrow e^{+} e^{-}\right)$, with quasireal photon beam. TCS is the time-reversal symmetric process to DVCS: the incoming photon is real and the outgoing photon has large timelike virtuality. In TCS, the virtuality of the outgoing photon $Q^{\prime 2} \equiv M^{2}$, where $M$ is the invariant mass of the lepton pair, sets the hard scale. In the regime $-t / Q^{\prime 2} \ll 1$, where $t$ is the squared momentum transfer to the target proton, the factorization theorem [19] applies: it separates the hard scattering process $\left(\gamma q \gamma^{*}\right)$, described via perturbation theory, from the soft dynamics encoded in GPDs (see Fig. 1, left). The TCS amplitude can then be expressed as a convolution of the hard scattering amplitude with GPDs, appearing in Compton form factors (CFFs). At leading order in $\alpha_{s}$, the CFF for the GPD $H$ is defined in Ref. [15] using the notations of Refs. [4,5] as

$\mathcal{H}(\xi, t)=\int_{-1}^{1} d x H(x, \xi, t)\left(\frac{1}{\xi-x+i \epsilon}-\frac{1}{\xi+x+i \epsilon}\right)$, 

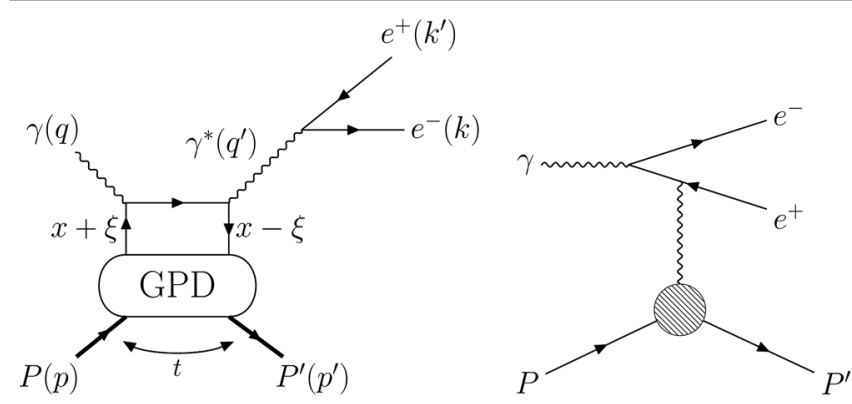

FIG. 1. Left: handbag diagram of the TCS process. Right: diagram of the $\mathrm{BH}$ process. $t=\left(p-p^{\prime}\right)^{2}$ is the squared fourmomentum transfer between the initial and final protons, $Q^{\prime 2}=$ $\left(k+k^{\prime}\right)^{2}$ is the invariant mass of the lepton pair, and $Q^{2}=-q^{2}$ is the virtuality of the real photon. $\xi=Q^{\prime 2} /\left(2\left(s-m_{p}^{2}\right)-Q^{\prime 2}\right)$ is the momentum imbalance of the struck quark, $s$ is the squared center-of-mass energy, and $m_{p}$ is the proton mass. $x$ is the average momentum fraction of the struck quark.

where $x, \xi$, and $t$ are defined in Fig. 1. Similar equations apply to the other GPDs $E, \tilde{E}$, and $\tilde{H}$. With a beam of circularly polarized photons, TCS can access both the real and imaginary parts of the CFFs [16].

As in DVCS, the Bethe-Heitler $(\mathrm{BH})$ process, which can be computed in a quasi-model-independent way, contributes to the same final state (see Fig. 1, right). The cross section for exclusive lepton pair photoproduction on the proton can be expressed as

$$
\sigma\left(\gamma p \rightarrow p^{\prime} e^{+} e^{-}\right)=\sigma_{\mathrm{BH}}+\sigma_{\mathrm{TCS}}+\sigma_{\mathrm{INT}},
$$

where INT stands for the TCS-BH interference term. As presented in Refs. $[15,16]$, the BH contribution dominates over the TCS in the total cross section by 2 orders of magnitude in the kinematic range accessible at Jefferson Lab (JLab). Therefore, the best practical way to access GPDs with the TCS reaction is to measure observables giving access to the TCS-BH interference. At leading order and leading twist in QCD, $\sigma_{\mathrm{INT}}$ can be expressed as a linear combination of GPD-related quantities [15],

$$
\begin{aligned}
\frac{d^{4} \sigma_{\mathrm{INT}}}{d Q^{\prime 2} d t d \Omega}= & A \frac{1+\cos ^{2} \theta}{\sin \theta} \\
& \times\left[\cos \phi \operatorname{Re} \tilde{M}^{--}-\nu \sin \phi \operatorname{Im} \tilde{M}^{--}\right],
\end{aligned}
$$

where

$$
\tilde{M}^{--}=\left[F_{1} \mathcal{H}-\xi\left(F_{1}+F_{2}\right) \tilde{\mathcal{H}}-\frac{t}{4 m_{p}^{2}} F_{2} \mathcal{E}\right]
$$

$A$ is a kinematic factor given in Ref. [15], $\phi$ and $\theta$ are defined in Fig. 2, $\Omega$ is the solid angle defined by $\theta$ and $\phi, \nu$ is the circular polarization of the photon beam (equal to +1 for right-handed and -1 for left-handed polarization), $m_{p}$ is the proton mass, $F_{1}$ and $F_{2}$ are the electromagnetic form

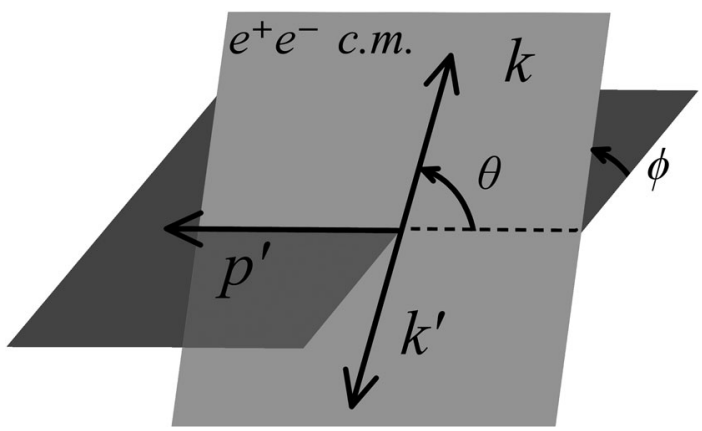

FIG. 2. Relevant angles for TCS. $\phi$ and $\theta$ are, respectively, the angle between the leptonic plane (defined by the outgoing leptons momenta $k$ and $k^{\prime}$ ) and the hadronic plane (defined by the incoming and outgoing proton momenta $p$ and $p^{\prime}$, defined in Fig. 1), and the angle between the electron and the recoiling proton in the leptons center-of-mass frame.

factors, and $\mathcal{H}, \tilde{\mathcal{H}}$, and $\mathcal{E}$ are the TCS CFFs of the $H, \tilde{H}$, and $E$ GPDs, respectively, which are given in Eq. (1). As the coefficients of $\tilde{\mathcal{H}}$ and $\mathcal{E}$ in Eq. (4) are suppressed, especially in the kinematics covered at JLab, measuring unpolarized and polarized observables linked to the TCS-BH interference cross section accesses mainly, respectively, the real and the imaginary parts of the $\mathcal{H}$ CFF.

In this Letter, two TCS observables were measured for the first time: the photon polarization asymmetry $A_{\odot U}$ and the forward-backward (FB) asymmetry $A_{\mathrm{FB}} . A_{\odot U}$ is proportional to the $\sin \phi$ moment of the polarized interference cross section and allows access to the imaginary part of $\mathcal{H}$. $A_{\mathrm{FB}}$, defined as

$$
A_{\mathrm{FB}}(\theta, \phi)=\frac{d \sigma(\theta, \phi)-d \sigma\left(180^{\circ}-\theta, 180^{\circ}+\phi\right)}{d \sigma(\theta, \phi)+d \sigma\left(180^{\circ}-\theta, 180^{\circ}+\phi\right)},
$$

projects out the $\cos \phi$ moment of the unpolarized cross section, proportional to the real part of the CFF $\mathcal{H}$ [20]. Both $A_{\odot U}$ and $A_{\mathrm{FB}}$ are zero if only $\mathrm{BH}$ contributes to the $\gamma p \rightarrow p^{\prime} \gamma^{*}$ cross section. Furthermore, it was shown in Ref. [21] that the QED radiative corrections are negligible for both of these observables.

The experiment was carried out in Hall B at JLab, using a 10.6-GeV electron beam, impinging on a 5-cm-long liquidhydrogen target placed at the center of the solenoid magnet of CLAS12 [22]. Potential quasireal photoproduction events $\left(e p \rightarrow p^{\prime} e^{+} e^{-} X\right)$ were selected requiring one electron, one positron, and one proton. The trajectories of charged particles, bent by the CLAS12 torus and solenoid magnetic fields, were measured by the drift chambers and in the central vertex tracker, providing their charge and momentum. The leptons were identified combining the information from the high-threshold Cherenkov counters and the forward electromagnetic calorimeter (ECAL) [23]. Leptons with momenta below $1 \mathrm{GeV}$ were removed to eliminate poorly reconstructed tracks in the forward 
detector. The background due to positive pions in the positron sample was minimized using a neural-networkbased multivariate analysis of the transverse and longitudinal profiles of showers in the ECAL. The protons were identified by analyzing the velocity of positive tracks measured by the time-of-flight systems as a function of their momentum. The momenta of the protons were corrected for energy loss in the detector materials using Monte Carlo (MC) simulations. Data-driven corrections were included to account, in the case of the leptons, for radiative losses and, in the case of protons, for detector-dependent momentum shifts not accounted by the simulation.

Once the $p^{\prime} e^{+} e^{-}$events were selected, exclusivity selection criteria were applied to ensure kinematics in the quasireal photoproduction regime [20]. The 4-momenta of the scattered electron and initial quasireal photon were determined via energy-momentum conservation from the measured 4-momenta of the final-state particles. Then the mass and the transverse momentum fraction $P_{t} / P$ of the scattered electron were constrained to be close to zero $\left(P_{t} / P<0.05,\left|M^{2}\right|<0.4 \mathrm{GeV}^{2}\right)$. These criteria ensure that the virtuality of the incoming photon is low $\left(Q^{2}<0.15 \mathrm{GeV}^{2}\right)$. The invariant mass spectrum of the outgoing lepton pair after exclusivity selection is shown in Fig. 3. The vector meson resonances decaying into an electron-positron pair are clearly visible. 2921 events with invariant mass between 1.5 and $3 \mathrm{GeV}$ were selected to measure the TCS observables. In this region, the factorization condition $-t / Q^{\prime 2} \ll 1$ needed for the GPD formalism to apply is fulfilled. In Fig. 3, the experimental invariant mass distribution is compared with $\mathrm{BH} \mathrm{MC}$ events. The good agreement between the two distributions rules out the possible contamination of the data by high mass meson

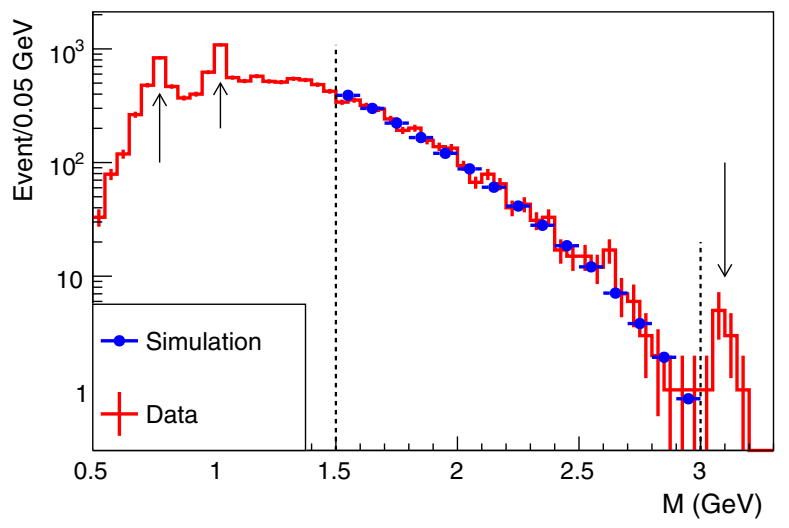

FIG. 3. Invariant mass of the electron-positron pairs. The indicated peaks correspond to the $\rho_{0} / \omega, \phi$, and $J / \psi$ mesons. The TCS events are selected in the 1.5-3-GeV mass range (within the dotted vertical lines) and are compared to $\mathrm{MC}$ simulation of $\mathrm{BH}$ events. The simulation is normalized to the total number of events. The data-simulation bin-by-bin ratio agrees at the $15 \%$ level. resonances decaying into $e^{+} e^{-}$pairs [e.g., $\rho(1450)$ and $\rho(1700)]$.

$A_{\odot U}$ was computed in four $t$ bins. The circular polarization of the quasireal photon was inferred from the longitudinal polarization of the electron it was radiated from. An electron polarized (with polarization $P_{b}$ ) in the direction (opposite) of the beam emits a right(left)-handed circularly polarized photon, with a transferred polarization $P_{\text {trans }}$ that can be calculated analytically [24] for each event. Taking advantage of the polarization transfer, the asymmetry $A_{\odot U}$, integrated over $\theta$, is measured as

$$
A_{\odot U}\left(-t, E_{\gamma}, M ; \phi\right)=\frac{1}{P_{b}} \frac{N^{+}-N^{-}}{N^{+}+N^{-}},
$$

where the number of events with reported positive and negative electron helicity in each bin is corrected by the acceptance and efficiency of CLAS12 (Acc) for the $\gamma p \rightarrow$ $p^{\prime} e^{+} e^{-}$reaction and by the polarization transfer as

$$
N^{ \pm}=\sum \frac{1}{\text { Acc }} P_{\text {trans }}
$$

Acc was estimated using the CLAS12 GEANT-4 [25] simulations framework [26]. A MC sample of $3.6 \times 10^{7}$ events was used. The acceptance was calculated in a fivedimensional grid of $\left(-t, E_{\gamma}, Q^{\prime 2}, \theta, \phi\right)$ bins. In a given bin, the acceptance is defined as the number of events reconstructed in this bin divided by the number of events generated in this bin. Low-occupancy bins, yielding an acceptance below 5\% and with a relative uncertainty greater than $50 \%$, were discarded from the analysis.

The obtained $\phi$ distributions of Eq. (6) are shown in Fig. 4 and are fitted with a sinusoidal function. In Fig. 5, the
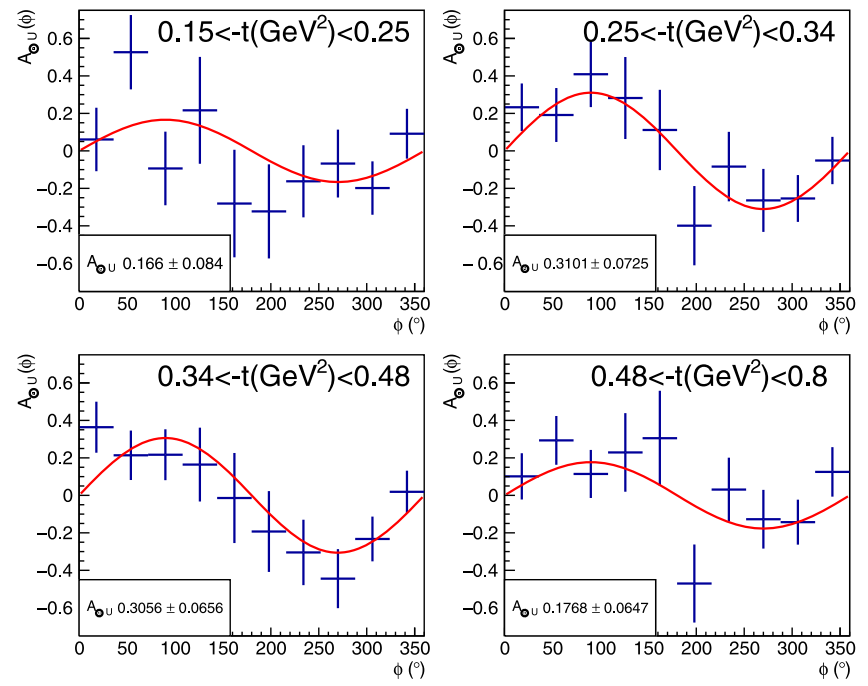

FIG. 4. $A_{\odot U}$ as a function of $\phi$ for the four $t$ bins used in this analysis. The sine fit function is superimposed. The amplitude of the fit $A_{\odot U}$ is plotted as a function of $-t$ in Fig. 5. 


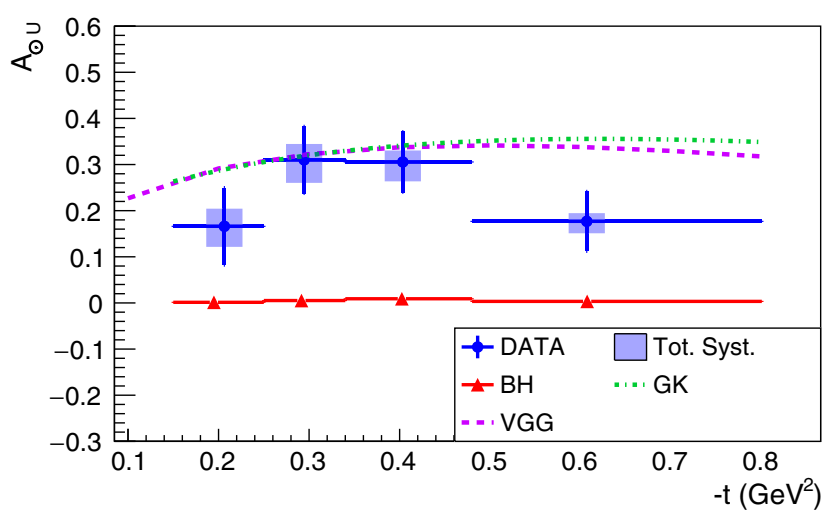

FIG. 5. $A_{\odot U}$ as a function of $-t$ at the averaged kinematic point $E_{\gamma}=7.29 \pm 1.55 \mathrm{GeV} ; M=1.80 \pm 0.26 \mathrm{GeV}$. The errors on the averaged kinematic point are the standard deviations of the corresponding distributions of events. The blue data points are represented with statistical error bars, horizontal bin widths, and shaded total systematic uncertainty. Red triangles show the asymmetry computed for simulated $\mathrm{BH}$ events. The dashed and dash-dotted lines are the predictions of, respectively, the VGG [27-30] and the GK [31-33] models, evaluated at the average kinematics.

$t$ dependence of the amplitude of the sinusoidal modulation is presented.

In-depth systematic checks were performed. Seven sources of systematic uncertainties were studied: the uncertainties associated with the binning of the acceptance corrections and with the rejection of low-acceptance bins; the uncertainties from the MC model used to calculate the acceptance and the related efficiency corrections; the systematic shifts induced by the identification procedure of protons and positrons; the impact of the variation of the exclusivity selection criteria. For each source of systematic uncertainty and for each bin, a value of systematic shift was added in quadrature after a smoothing procedure. This procedure was necessary to avoid the large bin-to-bin fluctuations of the systematic uncertainties due to the low statistics. The total systematic uncertainties are always smaller than the statistical uncertainties, typically by more than $50 \%$. The main contribution to the systematic uncertainties comes from the exclusivity selection.

In Figs. 4 and 5, a clear photon beam polarization asymmetry is observed. This arises from the BH-TCS interference, as the expected asymmetry for the $\mathrm{BH}$ contribution only, which was estimated using MC simulation, is zero. The photon polarization asymmetries were compared to predictions of the Vanderhaeghen-GuichonGuidal (VGG) model (based on a double-distribution (DD) parametrization with Regge-like $t$ dependence) [27-30] and of the Goloskokov-Kroll (GK) model (based on a DD parametrization with $t$ dependence expressed in the forward limit) [31-33] computed within the PARTONS framework [34]. Both of these calculations were performed at leading order in $\alpha_{s}$, which is a reasonable approximation in our kinematics, while QCD corrections have been shown to be quite important at lower values of $\xi$ [35-37]. The measured values [20] are in approximate agreement with the predictions of GPD-based models, while BH-only calculations show no asymmetry. This observation validates the application of the GPD formalism to describe TCS data and hints at the universality of GPDs, as the VGG and GK models also describe well the 6-GeV DVCS data from JLab [38].

Using the same dataset, $A_{\mathrm{FB}}$ was measured for four $t$ bins, integrating over all other kinematic variables due to the limited statistics of the analysis [20]. The angular coverage of CLAS12 allows one to measure $A_{\mathrm{FB}}$ only in a limited angular range. Thus, the forward and backward angles $\left(\phi_{F}, \theta_{F}, \phi_{B}=180^{\circ}+\phi_{F}\right.$ and $\left.\theta_{B}=180^{\circ}-\theta_{F}\right)$ were extracted in a forward region defined by $-40^{\circ}<\phi_{F}<40^{\circ}$, $50^{\circ}<\theta_{F}<80^{\circ}$ and in a corresponding backward region defined by $140^{\circ}<\phi_{B}<220^{\circ}, 100^{\circ}<\theta_{B}<130^{\circ}$. The value of $A_{\mathrm{FB}}$ was computed, for each $-t$ bin, as

$$
A_{\mathrm{FB}}=\frac{N_{F}-N_{B}}{N_{F}+N_{B}},
$$

where $N_{F / B}$ are the number of events in the forward and backward angular bins, corrected by the acceptance and the bin volume. The bin volume correction accounts for the difference in coverage between the forward and the backward directions, which could induce false asymmetries. This correction assumes that the cross section of the TCS reaction is constant within the volume of the forward (respectively, backward) bin and that it can be estimated only by measuring it in the volume covered by the acceptance of CLAS12. These approximations were accounted for in the systematic uncertainties by computing $A_{\mathrm{FB}}$ with $\mathrm{BH}$-weighted simulated events. The difference between the expected vanishing asymmetry and the obtained value was assigned as a systematic uncertainty.

Figure 6 shows $A_{\mathrm{FB}}$ for $1.5<M<3 \mathrm{GeV}$. In order to explore the dependence on the hard scale of the FB

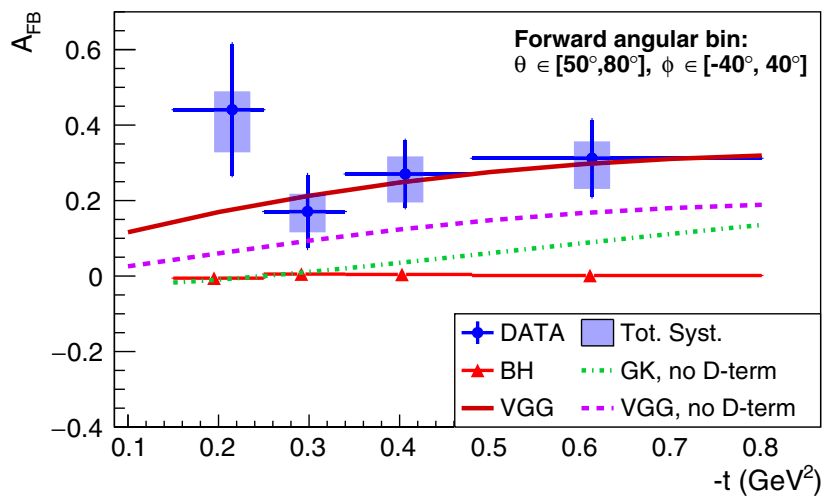

FIG. 6. FB asymmetry as a function of $-t$ at the average kinematics $E_{\gamma}=7.23 \pm 1.61 \mathrm{GeV} ; M=1.81 \pm 0.26 \mathrm{GeV}$. The solid line shows the model predictions of the VGG model with $D$ term (from Ref. [39]) evaluated at the average kinematic point. The other curves are defined in the caption of Fig. 5. 


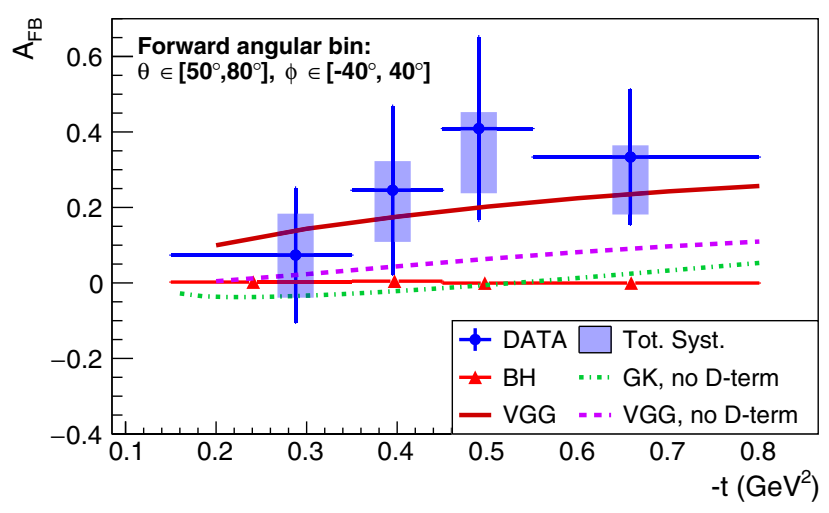

FIG. 7. FB asymmetry as a function of $-t$ at the average kinematics $E_{\gamma}=8.13 \pm 1.23 \mathrm{GeV} ; M=2.25 \pm 0.20 \mathrm{GeV}$. The curves are defined in the captions of Figs. 5 and 6.

asymmetry, it was extracted separately for the invariant mass region between 2 and $3 \mathrm{GeV}$ as shown in Fig. 7. The asymmetries in both mass regions are not comparable with the zero asymmetry predicted if only the $\mathrm{BH}$ process was contributing to the total cross section. This confirms that the TCS diagram contributes to the $\gamma p \rightarrow p^{\prime} e^{+} e^{-}$cross section. These results were compared with model predictions and seem to be better described by the VGG model when the $D$-term (taken from Ref. [39]) is included, although the error bars are still too large to completely rule out the case without the $D$-term. The $D$-term, a poorly known element of GPD parametrizations that appears as a subtraction term in dispersion relations of DVCS amplitudes, has recently gained relevance for its links to the mechanical properties of the nucleon [40-43]. The GK model predictions largely underestimate the asymmetry in both mass regions. This could be explained by the absence of the $D$-term in this prediction, although the GK model differs also from the VGG model without the $D$-term. The comparison was also done in the high-mass region in Fig. 7. In this region, where factorization-breaking terms are more strongly suppressed, the previous conclusion stands, supporting the interpretation in terms of GPDs and the importance of the $D$-term in their parametrization.

In summary, we reported in this Letter the first ever measurement of TCS on the proton. The photon circular polarization and forward-backward asymmetries were measured. The nonzero asymmetries provide strong evidence for the contribution of the quark-level mechanisms parametrized by GPDs to the cross section of this reaction. The comparison of the measured polarization asymmetry with model predictions points toward the interpretation of GPDs as universal functions. The reported results on the FB asymmetry open a new promising path toward the extraction of the real part of $\mathcal{H}$ and ultimately to a better understanding of the internal pressure of the proton via the extraction of the $D$-term. Future measurements of TCS at JLab will provide a wealth of data to be included in the ongoing fitting efforts to extract CFFs [44-47]. In particular, TCS measurements should have a strong impact in constraining the real part of CFFs [48] and in the determination of the $D$-term that relates to the gravitational form factor of the nucleon. A comparison of these results with possible measurements of TCS at the Electron Ion Collider [49] and in ultraperipheral collisions at the LHC [50] could provide a better understanding of the behaviour of the CFFs of TCS at low $x[36,37]$.

We thank Professors M. Vanderhaeghen, B. Pire, and P. Sznajder for the fruitful exchanges and discussions on the phenomenological aspects of this work and for providing us with the model predictions. We acknowledge the great efforts of the staff of the Accelerator and the Physics Divisions at Jefferson Lab in making this experiment possible. This work is supported in part by the U.S. Department of Energy, the National Science Foundation (NSF), the Italian Istituto Nazionale di Fisica Nucleare (INFN), the French Centre National de la Recherche Scientifique (CNRS), the French Commissariat pour l'Energie Atomique, the UK Science and Technology Facilities Council, the National Research Foundation (NRF) of Korea, the Helmholtz-Forschungsakademie Hessen für FAIR (HFHF), and the Ministry of Science and Higher Education of the Russian Federation. This project has received funding from the European Union's Horizon 2020 research and innovation programme under the grant agreement No. 824093. The Southeastern Universities Research Association (SURA) operates the Thomas Jefferson National Accelerator Facility for the U.S. Department of Energy under Award No. DE-AC0506OR23177.

*pierre.chata@orange.fr; Present address: INFN, Sezione di Genova, 16146 Genova, Italy.

†Present address: Mississippi State University, Starkville, Mississippi 39762-5167, USA.

*Present address: Argonne National Laboratory, Argonne, Illinois 60439, USA.

${ }^{\S}$ Present address: Università degli Studi di Brescia, 25123 Brescia, Italy.

[1] G. Altarelli, Phys. Rep. 81, 1 (1982).

[2] M. Diehl, Eur. Phys. J. A 52, 149 (2016).

[3] D. Müller, D. Robaschik, B. Geyer, F.-M. Dittes, and J. Hořejši, Fortschr. Phys. 42, 101 (1994).

[4] X. Ji, Phys. Rev. Lett. 78, 610 (1997).

[5] X. Ji, Phys. Rev. D 55, 7114 (1997).

[6] A. V. Radyushkin, Phys. Rev. D 56, 5524 (1997).

[7] A. V. Radyushkin, Phys. Lett. B 449, 81 (1999).

[8] M. Burkardt, Phys. Rev. D 62, 071503(R) (2000).

[9] S. Stepanyan et al. (CLAS Collaboration), Phys. Rev. Lett. 87, 182002 (2001).

[10] C. Muñoz Camacho et al. (Jefferson Lab Hall A Collaboration), Phys. Rev. Lett. 97, 262002 (2006). 
[11] F. X. Girod et al. (CLAS Collaboration), Phys. Rev. Lett. 100, 162002 (2008).

[12] E. Seder et al. (CLAS Collaboration), Phys. Rev. Lett. 114, 032001 (2015).

[13] S. Pisano et al. (CLAS Collaboration), Phys. Rev. D 91, 052014 (2015).

[14] H. S. Jo et al. (CLAS Collaboration), Phys. Rev. Lett. 115, 212003 (2015).

[15] E. Berger, M. Diehl, and B. Pire, Eur. Phys. J. C 23, 675 (2002).

[16] M. Boër, M. Guidal, and M. Vanderhaeghen, Eur. Phys. J. A 51, 103 (2015).

[17] P. Nadel-Turonski, T. Horn, Y. Ilieva, F. J. Klein, R. Paremuzyan, and S. Stepanyan, AIP Conf. Proc. 1182, 843 (2009).

[18] M. Boër, M. Guidal, and M. Vanderhaeghen, Eur. Phys. J. A 52, 33 (2016).

[19] J. C. Collins and A. Freund, Phys. Rev. D 59, 074009 (1999).

[20] See Supplemental Material at http://link.aps.org/supplemental/ 10.1103/PhysRevLett.127.262501 for a detailed description of the quasireal photoproduction reaction of lepton pairs, on the extraction of the real part of the Compton amplitude in the forward-backward asymmetry and for the numerical values of the obtained results.

[21] M. Heller, N. Keil, and M. Vanderhaeghen, Phys. Rev. D 103, 036009 (2021).

[22] V. D. Burkert et al., Nucl. Instrum. Methods Phys. Res., Sect. A 959, 163419 (2020).

[23] V. Ziegler et al., Nucl. Instrum. Methods Phys. Res., Sect. A 959, 163472 (2020).

[24] H. Olsen and L. C. Maximon, Phys. Rev. 114, 887 (1959).

[25] S. Agostinelli et al., Nucl. Instrum. Methods Phys. Res., Sect. A 506, 250 (2003).

[26] M. Ungaro et al., Nucl. Instrum. Methods Phys. Res., Sect. A 959, 163422 (2020).

[27] M. Vanderhaeghen, P. A. M. Guichon, and M. Guidal, Phys. Rev. Lett. 80, 5064 (1998).

[28] M. Vanderhaeghen, P. A. M. Guichon, and M. Guidal, Phys. Rev. D 60, 094017 (1999).
[29] M. Guidal, M. V. Polyakov, A. V. Radyushkin, and M. Vanderhaeghen, Phys. Rev. D 72, 054013 (2005).

[30] M. Guidal, H. Moutarde, and M. Vanderhaeghen, Rep. Prog. Phys. 76, 066202 (2013).

[31] S. V. Goloskokov and P. Kroll, Eur. Phys. J. C 42, 281 (2005).

[32] S. V. Goloskokov and P. Kroll, Eur. Phys. J. C 53, 367 (2008).

[33] S. V. Goloskokov and P. Kroll, Eur. Phys. J. C 65, 137 (2010).

[34] B. Berthou et al., Eur. Phys. J. C 78, 478 (2018).

[35] B. Pire, L. Szymanowski, and J. Wagner, Phys. Rev. D 83, 034009 (2011).

[36] D. Müller, B. Pire, L. Szymanowski, and J. Wagner, Phys. Rev. D 86, 031502(R) (2012).

[37] H. Moutarde, B. Pire, F. Sabatié, L. Szymanowski, and J. Wagner, Phys. Rev. D 87, 054029 (2013).

[38] R. Dupre, M. Guidal, and M. Vanderhaeghen, Phys. Rev. D 95, 011501(R) (2017).

[39] B. Pasquini, M. Polyakov, and M. Vanderhaeghen, Phys. Lett. B 739, 133 (2014).

[40] M. Polyakov, Phys. Lett. B 555, 57 (2003).

[41] V. D. Burkert, L. Elouadrhiri, and F. X. Girod, Nature (London) 557, 396 (2018).

[42] K. Kumerizčki, Nature (London) 570, E1 (2019).

[43] H. Dutrieux, C. Lorcé, H. Moutarde, P. Sznajder, A. Trawiński, and J. Wagner, Eur. Phys. J. C 81, 300 (2021).

[44] K. Kumerički, S. Liuti, and H. Moutarde, Eur. Phys. J. A 52 , 157 (2016).

[45] R. Dupré, M. Guidal, S. Niccolai, and M. Vanderhaeghen, Eur. Phys. J. A 53, 171 (2017).

[46] H. Moutarde, P. Sznajder, and J. Wagner, Eur. Phys. J. C 78, 890 (2018).

[47] H. Moutarde, P. Sznajder, and J. Wagner, Eur. Phys. J. C 79, 614 (2019).

[48] O. Grocholski, H. Moutarde, B. Pire, P. Sznajder, and J. Wagner, Eur. Phys. J. C 80, 171 (2020).

[49] R. A. Khalek et al., arXiv:2103.05419.

[50] B. Pire, L. Szymanowski, and J. Wagner, Phys. Rev. D 79, 014010 (2009). 